\title{
Antimicrobial Surveillance for Bacterial
}

\section{Uropathogens in Ha'il, Saudi Arabia: A Five-Year Multicenter Retrospective Study}

This article was published in the following Dove Press journal:

Infection and Drug Resistance

\author{
Abdulrahman S Bazaid (D) \\ Amir Saeed ${ }^{1,2}$ \\ Abdulaziz Alrashidi ${ }^{\prime}$ \\ Abdulkarim Alrashidi ${ }^{1}$ \\ Khalid Alshaghdali' \\ Sahar A Hammam ${ }^{3}$ \\ Talal Alreshidi ${ }^{3}$ \\ Munif Alshammary ${ }^{3}$ \\ Abdullah Alarfaj (D) \\ Rawan Thallab ${ }^{4}$ \\ Abdu Aldarhami $\mathbb{D D}^{5}$ \\ 'Department of Clinical Laboratory \\ Sciences, College of Applied Medical \\ Sciences, University of Ha'il, Ha'il, Saudi \\ Arabia; ${ }^{2}$ Department of Medical \\ Microbiology, Faculty of Medical \\ Laboratory Sciences, University of \\ Medical Sciences \& Technology, \\ Khartoum, Sudan; ${ }^{3} \mathrm{Ha}$ 'il Maternity \\ Hospital, Ha'il, Saudi Arabia; \\ ${ }^{4}$ Department of Statistics, Faculty of \\ Science, King Abdulaziz University, \\ Jeddah, Saudi Arabia; ${ }^{5}$ Department of \\ Medical Microbiology, Qunfudah Faculty \\ of Medicine, Umm Al-Qura University, \\ Al-Qunfudah, 288I4, Saudi Arabia
}

Correspondence: Abdulrahman S Bazaid Department of Clinical Laboratory

Sciences, College of Applied Medical

Sciences, University of Ha'il, Ha'il, Saudi

Arabia

Tel +966-165358200

Email ar.bazaid@uoh.edu.sa
Objective: Urinary tract infections (UTIs) are among the most common infections that require antibiotic intervention. Antibiotic surveillance programs are crucial to assess resistance patterns of microorganisms associated with UTIs and to tailor antibiotic therapy accordingly. Therefore, the aim of the current study is to investigate the prevalence of uropathogens and their antimicrobial susceptibility patterns in Ha'il region.

Methods: We conducted a retrospective study in two main hospitals in Ha'il over a 5-year period (January 2015 to December 2019). Laboratory reports and clinical data of patients with a positive urine culture $\left(\geq 10^{5} \mathrm{CFU} / \mathrm{mL}\right)$ were included in the study.

Results: A total of 428 patients were included in this study. The majority of positive cultures belonged to female patients $(94.4 \%)$, adults $(76.9 \%)$ and outpatients $(74.3 \%)$. Generally, Escherichia coli was the most common pathogen (45\%), followed by Klebsiella pneumoniae $(17 \%)$ and Staphylococcus aureus (12\%). Extended-spectrum $\beta$-lactamase (ESBL) strains of E. coli $(15.7 \%)$ and $K$. pneumoniae $(19.7 \%)$ showed resistance to most tested antibiotics, while almost all Gram-negative uropathogens including ESBL strains presented low resistance to cefoxitin, imipenem and meropenem. Most strains of Staphylococcus aureus and Enterococcus faecalis were resistant to trimethoprim/sulfamethoxazole including methicillinresistant Staphylococcus aureus (MRSA) and vancomycin-resistant Enterococcus faecalis (VRE), whereas the activity of linezolid was monitored against almost all different strains of Gram-positive uropathogens.

Conclusion: This surveillance study which was conducted over a 5-year period in the Ha'il region revealed that the most common UTI-associated pathogen was E. coli. Based on the current sensitivity profiles obtained from this surveillance, carbapenems and linezolid can be considered as a first therapeutic choice treating UTIs in Ha'il caused by Gramnegative and positive uropathogens, respectively. Frequent targeted surveillance programs for antibiotic-resistant pathogens and their susceptibility profiles are crucial to enable tailored empirical treatment for patients.

Keywords: surveillance, antimicrobial resistance, AMR, uropathogens, Saudi Arabia, drugresistant bacteria

\section{Introduction}

Urinary tract infections (UTIs) are a very common type of infectious disease that requires immediate treatment to avoid serious complications, such as pyelonephritis. ${ }^{1}$ Approximately $60 \%$ of women will develop at least one symptomatic UTI during their lifetime, with higher rates of infection observed among sexually active females. ${ }^{2}$ By contrast, men are less prone to community-associated 
UTIs and its complications, and this is attributable mainly to anatomical differences. ${ }^{2}$ Over 150 million people are diagnosed with UTIs worldwide annually, ${ }^{1}$ accounting for $8 \%$ and $2 \%$ of total reported infection cases in the United States $^{3}$ and France, ${ }^{4}$ respectively. UTIs account for almost $10 \%$ of all infections in the Kingdom of Saudi Arabia (KSA), making these infections the second most common cause of admission to emergency departments in KSA. ${ }^{5}$ The economic cost of the treatment of UTIs in KSA is estimated to exceed $\$ 800,000$ annually in a single hospital. $^{6}$

UTIs can be caused by (Gram-positive or Gramnegative) bacteria, viruses or fungi, with more than $80 \%$ of UTIs being of bacterial origin. ${ }^{2,7}$ Most UTIs (75\%) are caused by Escherichia coli, ${ }^{7}$ followed by Klebsiella pneumoniae, Staphylococcus saprophyticus, Enterococcus faecalis and Proteus mirabilis. ${ }^{2}$ Multiple epidemiological studies have shown that antibiotic susceptibility profiles of the most predominant etiological microorganisms, including these bacteria, vary in relation to geographical location. ${ }^{8,9}$ This phenomenon is possibly influenced by the observation that different classes of antibiotics are used by different clinics in different regions based on their recommended guidelines for the treatment of UTIs. ${ }^{9-11}$ Thus, frequent local surveillance of antimicrobial resistance (AMR) should facilitate rational and local empirical use of drugs to treat UTIs. This would entail prescribing prophylactic antibiotics for uropathogens (UTI-causing bacteria) based on recent surveillance studies to ensure the eradication of the most likely causative bacterium, limit potential complications and shorten the treatment plan. $^{5}$

Action to control the spread of AMR is urgently required $^{12}$ as the prevalence of antibiotic resistance among uropathogens continues to increase. ${ }^{13-15}$ Consequently, multiple steps have been recommended, each having a significant role in controlling AMR, with antibiotic surveillance programs being 1 of the top 10 strategies to combat resistance. ${ }^{16}$ Antibiotic surveillance programs for microorganisms associated with UTIs have been routinely conducted in certain Saudi cities, including Makkah, ${ }^{17}$ Jeddah $^{18}$ and Riyadh. ${ }^{19}$ E. coli was previously determined as the most frequently identified bacterium from patients with UTIs in Riyadh. ${ }^{19}$ Isolated E. coli strains showed resistance to multiple antibiotics, including co-trimoxazole, ampicillin, ${ }^{19}$ cefoxitin and gentamicin, while almost half Staphylococcus aureus isolates were resistant to gentamicin. ${ }^{9}$ In addition, $74.4 \%$ of isolated Enterococcus faecium and $78.2 \%$ of Streptococcus agalactiae isolates were resistant to ampicillin, whereas $53.1 \%$ of Acinetobacter species displayed resistance to ciprofloxacin. ${ }^{9}$ A similar investigation revealed that the majority of isolated Gram-negative bacteria in Saudi Arabia are sensitive to co-trimoxazole and ciprofloxacin. ${ }^{20}$ Although the highlighted findings present some indication of resistance profiles in Saudi Arabia, there is still insufficient information about the prevalence of UTI-related bacteria and their antibiotic susceptibility profiles in the Ha'il region. Therefore, the aim of the current study was to measure the prevalence of infections by uropathogenic bacteria and their sensitivity patterns to currently used antibiotics in the province of Ha'il, KSA.

\section{Methods}

\section{Study Design}

A retrospective cohort study was conducted in the present work to report on bacteria associated with UTIs and their antibiotic resistance profiles from confirmed cases in the Ha'il region, KSA. Primary data were collected over a period of 5 years (January 2015 to December 2019) in two main hospitals in Ha'il, North of Saudi Arabia: a general hospital (Ha'il Maternity Hospital) with a capacity of 300 beds, and a private hospital, with a capacity of 150 beds.

\section{Study Cohort}

Single (pure culture) bacterial species identified from positive urine cultures collected between 2015 and 2019 were included in this study. Positive urine cultures were designated as those with $\geq 10^{5}$ colony forming units (CFU) per milliter of a single identified bacterial species. A midstream urine sample was collected by the adult patients themselves using dedicated sterile containers, while samples from infant patients were collected by nurses into sterile urine bags. For patients with more than one urine culture, only the first reported episode was included in this study due to unavailability of guidelines to distinguish between multiple cultures. Cultures with polymicrobial growth ( $>1$ species), low number of colonies $\left(<10^{5} \mathrm{CFU} / \mathrm{mL}\right)$ and those belonging to patients on antibiotic drugs were excluded from this investigation. Patients with positive cultures were classified as patients with UTIs and were categorised into different groups based on their age, gender and admission status (in or outpatient). 


\section{Bacterial Identification and Antibiotic Sensitivity Testing}

Urine samples were cultured on defibrinated sheep blood/ cystine lactose electrolyte deficient agar (CLED) plates (Oxoid, Basingstoke, UK), using a calibrated loop of 1 $\mu \mathrm{L}$, and were then incubated for $24-48$ hours at $37^{\circ} \mathrm{C}$. Subsequently, isolated bacteria were Gram stained and classified as Gram-positive cocci (GPC) or Gramnegative rods (GNR), followed by full identification and antibiotic sensitivity testing using the VITEK system (bioMerieux, UK). ${ }^{21}$ Obtained data were analysed and interpreted according to the guidelines of the Clinical Laboratory Standards Institute (CLSI). Susceptibility of recovered Gram-negative UTI-related bacteria was evaluated against a panel of antibiotics, including amikacin, ampicillin, cefepime, ceftazidime, cefoxitin, ciprofloxacin, gentamicin, imipenem, meropenem, nitrofurantoin, piperacillin, tigecycline and trimethoprim/sulfamethoxazole. For Gram-positive UTI-associated bacteria, assessment was conducted against ampicillin, cephalothin, clindamycin, levofloxacin, linezolid, moxifloxacin, nitrofurantoin, penicillin, tigecycline, trimethoprim/sulfamethoxazole and vancomycin. Sensitivity testing of ceftazidime against Gram-negative rods was conducted to determine extendedspectrum $\beta$-lactamase (ESBL) producing strains, while methicillin-resistant Staphylococcus aureus (MRSA) strains were identified by the use of cefoxitin. This is because resistant strains of Gram-negative bacilli and $S$. aureus to ceftazidime and cefoxitin are considered as $\mathrm{ESBL}^{22}$ and MRSA stains, ${ }^{23}$ respectively.

\section{Statistical Analysis}

Demographics of the patients, urine culture reports and antibiotic resistance profiles were electronically retrieved from the hospitals' medical records, and the data were presented as the numbers of cases (or resistant cases) for each isolated species and the percentages to total. Statistical analysis was performed using SPSS software version 16.0 (IBM Corporation, NY, USA).

\section{Ethical Approval}

This study was approved by the Ethics Committee at the University of Ha'il, Saudi Arabia (approval number H-2020-048). Patient informed consent was not required and waived by the Ethics Committee at the University of Ha'il because this is a retrospective study and there was no interaction with patients. Patient privacy and confidentiality of data were maintained in accordance with the Declaration of Helsinki.

\section{Results}

\section{Demographic Characteristics of the Study Cohort}

A total of $428(19.6 \%)$ out of 2185 samples were determined as positive urine cultures during the study period (January 2015 to December 2019) in two hospitals in Ha'il, Saudi Arabia. The majority of these positive cultures belonged to female patients $(94.4 \%)$, adolescent and adult aged $13-65$ years $(76.9 \%)$ and outpatients $(74.3 \%)$. $E$. coli was the predominant bacterium among different age and admission (in-patient or out-patient) groups of female patients, whereas $S$. aureus was the most commonly detected uropathogen in males (Table 1).

\section{Isolated UTI Causative Bacteria}

The total number of annually recovered UTI-associated bacteria varied over the 5 years of investigation; the lowest monitored number of isolates $(n=59)$ was recorded in 2016, whereas in the year 2018, the highest number of isolates $(n=108)$ was reported. Gram-negative bacilli were determined as the main class of bacteria associated with UTIs in the study cohort between 2015 and 2019, with $E$. coli being the most common uropathogen among isolated bacteria (45\% in 2015, and lower in subsequent years to around $35 \%$ in 2019). K. pneumoniae accounted for about $17 \%$ of UTIs annually (2015-2019), followed by $P$. aeruginosa (at 5\%). Gram-positive cocci, including $S$. aureus, E. faecalis and $S$. agalactiae, were the cause of approximately $12 \%, 6 \%$ and $5 \%$ of UTIs, respectively, in 2015. Almost 4\% of isolated S. aureus in 2018 were MRSA strains, although there was no record of any MRSA strain in other years of this investigation. The proportion of UTIs caused by S. aureus increased from 5\% in 2015 to $12 \%$ in 2019. Other species with lower frequencies of occurrence were $P$. mirabilis, $A$. baumannii and E. cloacae (Figure 1). In relation to ESBL production, $15.7 \%$ and $19.7 \%$ of all E. coli and $K$. pneumoniae isolates, respectively, were resistant to ceftazidime. The highest percentage of ESBL-producing E. coli (23.7\%) and $K$. pneumoniae (40\%) over the period of the investigation (2015-2019) was recorded in 2015, which dropped to $17.6 \%$ and $16.7 \%$, respectively, in 2019. Moreover, ESBLproducing $P$. mirabilis was only observed in 2018 (2 out of 3 P. mirabilis isolates during the study period). 


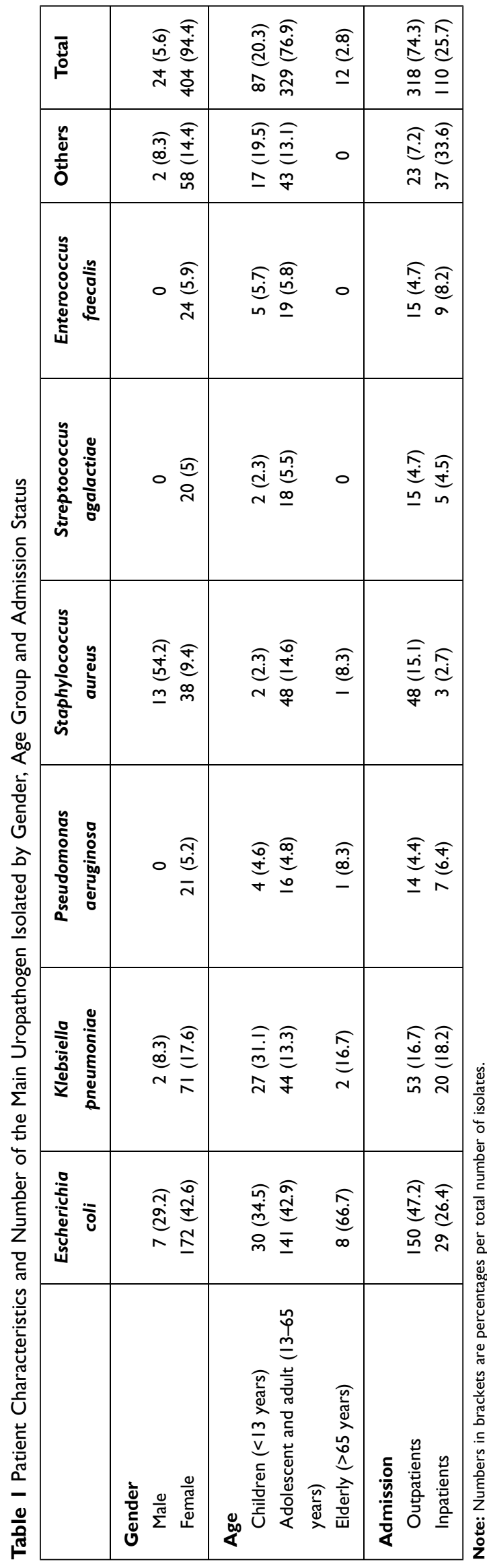

\section{Antibiotic Resistance Patterns}

Antimicrobial susceptibility testing of Gram-negative bacteria was conducted against 12 commonly used antibiotics to determine their resistance profiles. Non-ESBL producing strains of E. coli and K. pneumoniae showed high resistance to piperacillin and trimethoprim/sulfamethoxazole (Table 2). Likewise, the majority of non-ESBL producing $P$. mirabilis, $P$. aeruginosa and A. baumannii exhibited resistance to tigecycline and trimethoprim/sulfamethoxazole. Resistance profiles for ESBL-producing bacteria revealed that the majority of isolates were resistant to ampicillin, ciprofloxacin, gentamicin, nitrofurantoin, piperacillin, tigecycline and trimethoprim/sulfamethoxazole. ESBL and non-ESBL producing Gram-negative bacteria displayed limited levels of resistance to cefoxitin, imipenem and meropenem (Table 2).

Most of the identified Gram-positive bacteria exhibited limited resistance to the majority of tested antibiotics (Table 3). Almost third of isolated E. faecalis (7/24) were vancomycin-resistant $E$. faecalis (VRE) and presented a similar resistance pattern to non-VRE that were resistant to levofloxacin, clindamycin, trimethoprim/sulfamethoxazole and nitrofurantoin. Similarly, there was not any significant difference in the obtained sensitivity profiles among MRSA and methicillin-sensitive $S$. aureus (MSSA) strains and the majority of them were resistant to penicillin, trimethoprim/sulfamethoxazole and levofloxacin. Almost all Gram-positive uropathogens including MRSA and VER showed great sensitivity to linezolid. A quarter and just over one-sixth of $S$. agalactiae strains were resistant to trimethoprim/sulfamethoxazole and clindamycin, respectively (Table 3 ).

Resistance profiles of the three most common species associated with UTIs (E. coli, $P$. aeruginosa and K. pneumoniae) in Ha'il, Saudi Arabia, between 2015 and 2019 are surmised in Figure 2. Strains of E. coli showed a sharp increase in resistance to piperacillin and gentamicin (during the period 2015-2016), while the activity of these antibiotics led to resistance between $0 \%$ and $20 \%$ from 2017 onward. About $45 \%$ of $E$. coli isolates were resistant to piperacillin in 2017 , which decreased to below $30 \%$ by 2019 . During the study period, resistance of E. coli strains to cefoxitin and cefepime remained low (under 5\%).

In 2016, resistance of recovered K. pneumoniae strains to nitrofurantoin, gentamicin and amikacin was high in the range of $40-80 \%$ compared to other years of the study. 


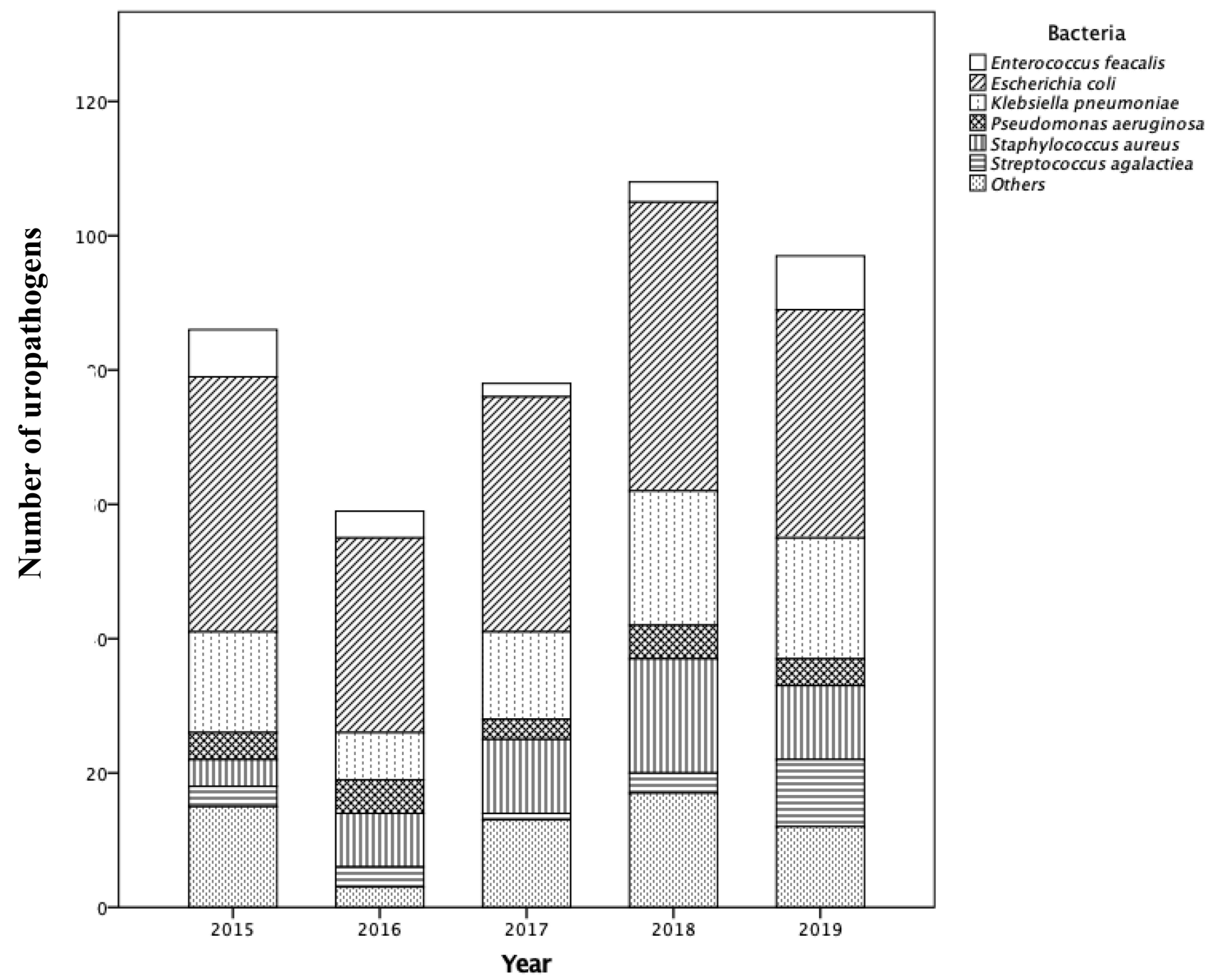

Figure I The number of monitored UTI-associated bacterial isolates from two hospitals in the Ha'il region of Saudi Arabia over five years (20I5-20I9). Others include Proteus mirabilis, Acinetobacter baumannii and Enterobacter cloacae.

Although the sensitivity of $K$. pneumoniae strains to these drugs during the following 4 years was notably high, a zero case of resistance to amikacin was reported in 2019. In addition, the activity of piperacillin towards strains of this bacterium increased gradually leading to $39 \%$ cases of resistance by 2019 compared to $6 \%$ in 2015. Resistance of K. pneumoniae strains to cefepime declined from $40 \%$ in 2015 to zero cases in 2017. Furthermore, resistance to cefepime in the following years (2017-2019) remained the same $(0 \%$ case of resistance).

Different resistance profiles of $P$. aeruginosa strains over the period of investigation (2015-2019) towards antibiotics were recorded. In 2015, the activity of trimethoprim/sulfamethoxazole against strains of this bacterium was completely absent, which was then appeared in the following years leading to about $50 \%$ resistant strains in 2019. There was zero case of resistance of $P$. aeruginosa in 2015 against piperacillin, while $66 \%$ resistant strains were monitored in 2017; however, it dropped to $25 \%$ by 2019. The activity of gentamicin against isolated of $P$. aeruginosa decreased gradually between 2015 and 2017 causing $25 \%$ and $66 \%$ resistant strains, followed by full effectiveness, no resistant isolate reported in 2019.

\section{Discussion}

The ongoing global rise of AMR among UTI-causing bacteria has reduced the effectiveness of antibiotics in clinical use. ${ }^{24}$ Regional monitoring programs (surveillance) for resistant bacteria are therefore crucial to tailoring suitable usage guidelines for antibiotics. Using clinical data based on local monitoring programs, the current study 

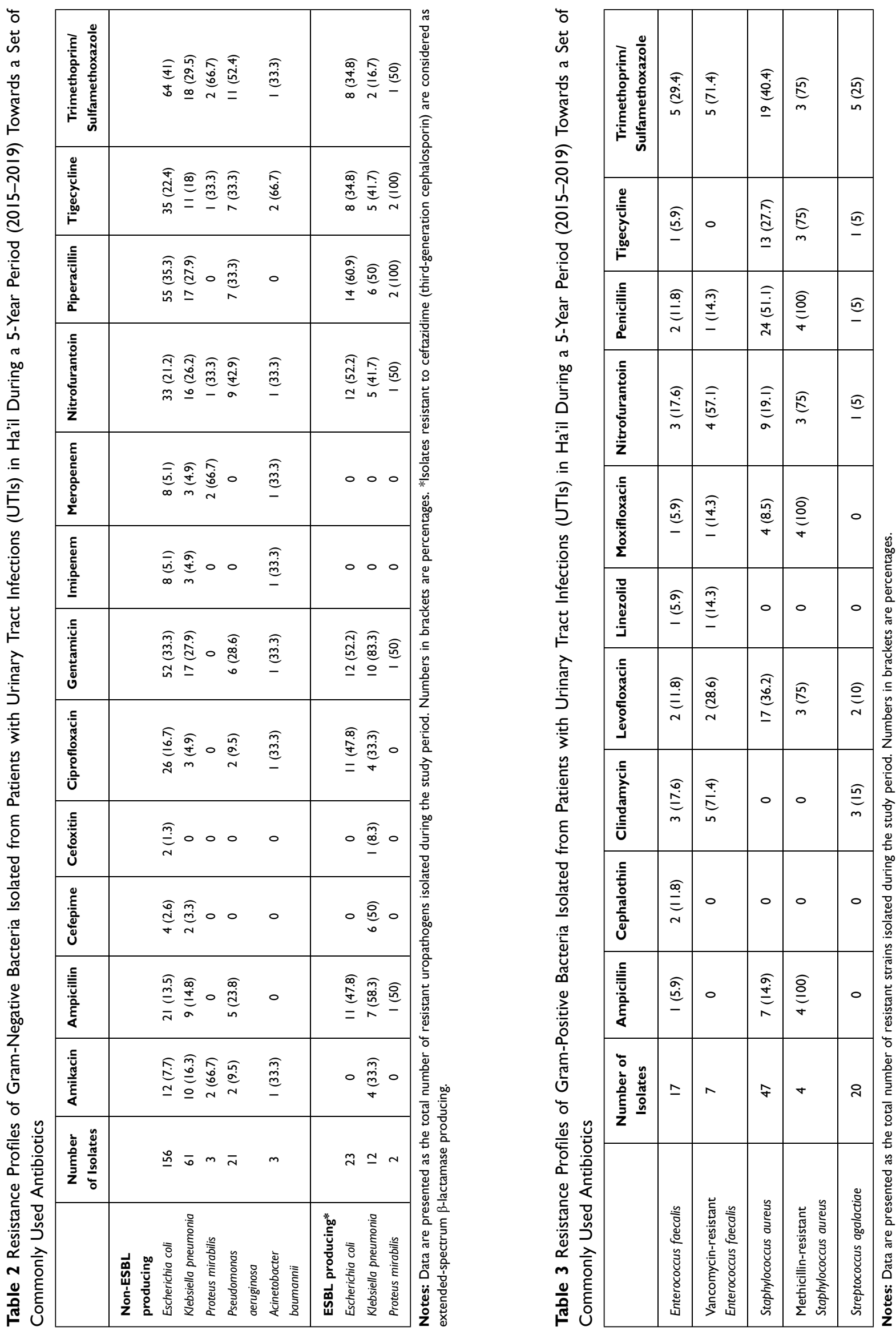
-Amikacin

Cefoxitin

- Imipenem

a Piperacillin
Ampicillin

- Ciprofloxacin

Meropenem

$\square$ Tigecycline
Cefepime

Gentamicin

Nitrofurantoin

Trimethoprim/sulfamethoxazole

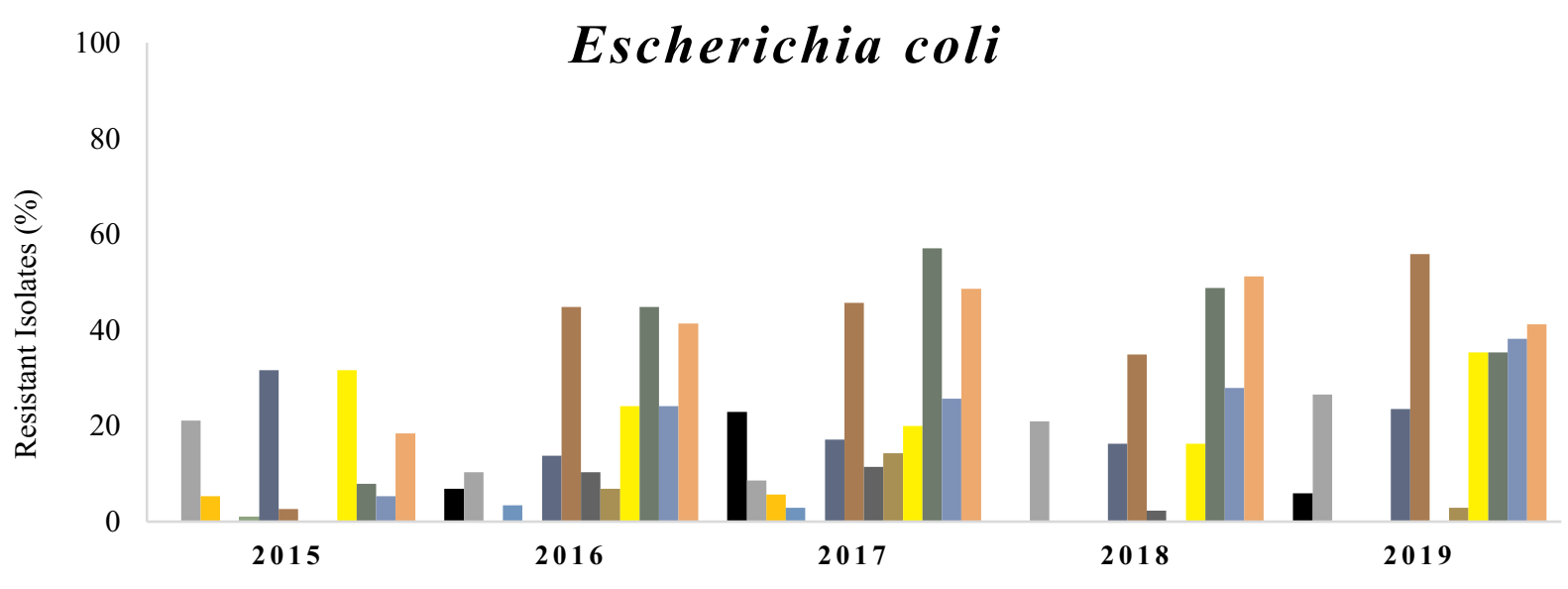

\section{Klebsiella pneumoniae}

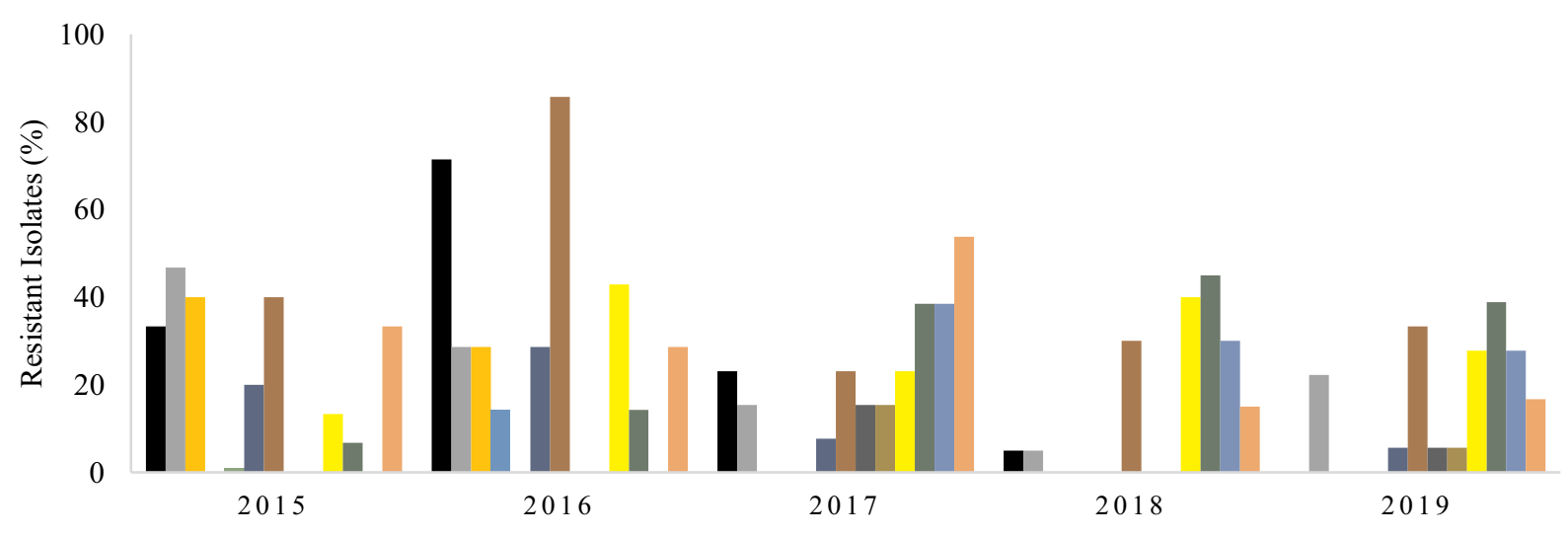

\section{Pseudomonas aeruginosa}

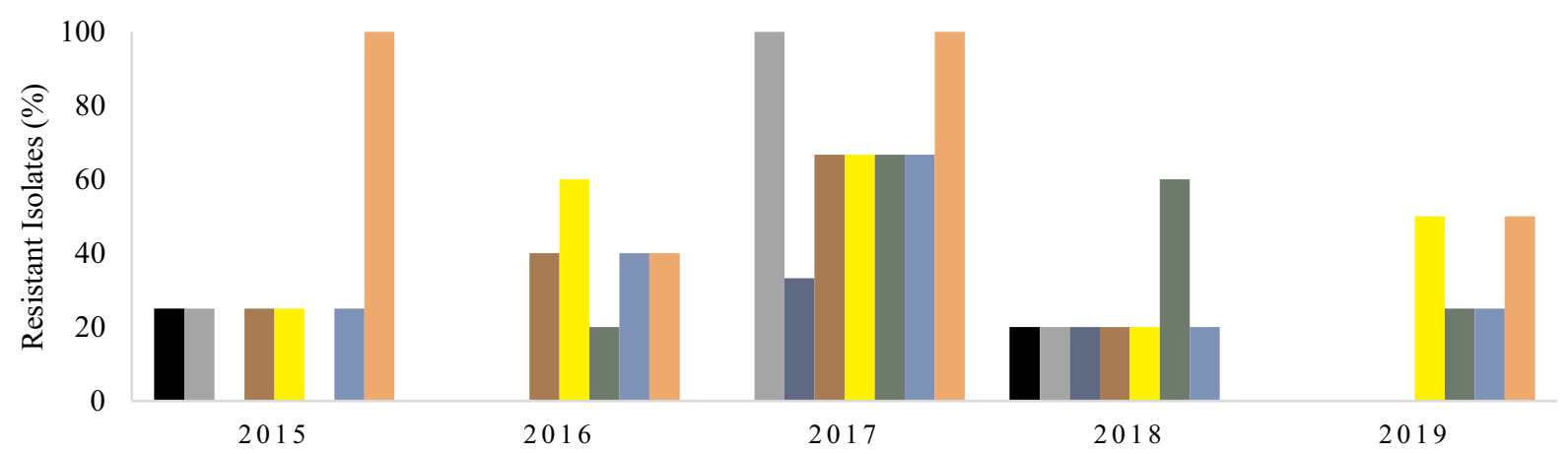

Figure 2 Annual percentage (as a percentage) of antibiotic resistance among the most commonly identified Gram-negative (Escherichia coli, Klebsiella pneumoniae and Pseudomonas aeruginosa) in patients with UTIs in Ha'il, Saudi Arabia, between 2015 and 2019. 
revealed the prevalence of uropathogens associated with UTIs and indicated their antibiotic resistance profiles against commonly used antibiotics between 2015 and 2019 in Ha'il, KSA.

The study cohort included 428 participants, and in agreement with previous studies, ${ }^{18,25}$ the majority of patients with UTIs were young females. This is unsurprising and is likely the result of the anatomy of the female urinary tract compared to their male counterparts, particularly the shorter female urethra and closer proximity to the anus. ${ }^{26}$ Thus, prescription and over-thecounter use of antibiotics to treat UTIs is widely observed in young females, which explains their high susceptibility to both recurrent and drug-resistant infections. $^{27}$ In agreement with $^{19,22}$ the current data showed that the majority of patients are adolescent and adults (13-65 years). However, in contrast, a recent report revealed an increase in UTI incidence among older females ( $>50$ years old), ${ }^{28}$ and this variation in age groups may be due to differences in the study cohort since part of the retrieved data of this study were collected from pregnant women.

Etiological agents of UTIs and their susceptibility/ resistance patterns differ based on geographical location, age and gender, and this observation highlights the importance of conducting frequent local surveillance programs with a wide range of patients to ensure appropriate treatment choices are available. ${ }^{25,27}$ Enterobacteriaceae were observed to be the predominant family of bacteria isolated from patients with UTIs in all age groups in Ha'il. This finding is in line with previous investigations in multiple countries, ${ }^{25,29}$ including Saudi Arabia, ${ }^{19,30}$ where E. coli was identified as the main causative bacterium of UTIs, followed by K. pneumoniae. ${ }^{30,31}$

Several studies reported high incidence of $P$. mirabilis in paediatric patients, which was not the case in the current study. P. mirabilis is commonly present in the preputial sac in boys, ${ }^{32}$ while the population of Saudi males are largely circumcised as part of religious practice, which should explain this variation between communities. Strains of E. coli accounted for $45 \%$ of recovered isolates in 2015 and this percentage dropped to about $35 \%$ in 2019. In the same period, there was a notable increase in the prevalence of $S$. aureus among patients with UTIs. Although $S$. aureus was previously reported to be among rarely isolated UTIcausing bacteria, ${ }^{33}$ it was recently reported as the causative agent responsible for about a third of UTIs in Nigeria. ${ }^{8}$ High prevalence of UTIs caused by $S$. aureus was previously linked with young females ${ }^{8}$ and this might be the reason behind the observed prevalence in this study since this group accounts for the majority of patients in Ha'il. Moreover, S. aureus was the main causative agent of UTI in males, which concurs with a previous report conducted in the United Kingdom. ${ }^{34}$

Certainly, the usage of antibiotics based on laboratory tests (antimicrobial susceptibility) should limit/control the spread of AMR ${ }^{24}$ However, since sensitivity testing takes about $24-48$ hours, ${ }^{35}$ there is a pressing need for guidelines for immediate empirical use of antibiotics for critical UTI cases prior to laboratory tests. ${ }^{36}$ Thus, an operational description of a set of empirically selected drugs would heavily rely on accessible, recent and local surveillance data on AMR since the resistance patterns are continuously changing over time and from place to another. ${ }^{8,36}$ In vitro antimicrobial sensitivity assays remain significantly important and should be frequently performed to identify any possible emergence of resistant strains. Most of the recovered non-ESBL producing Gram-negative isolates displayed high levels of resistance to piperacillin and trimethoprim/sulfamethoxazole. This resistance profile is similar to those monitored nationally in other regions of Saudi Arabia ${ }^{19,30,31}$ and worldwide ${ }^{37}$ for strains of $E$. coli and $K$. pneumoniae against trimethoprim/sulfamethoxazole. The emergence of highly resistant uropathogens, especially E. coli and $P$. aeruginosa, to trimethoprim/sulfamethoxazole is indeed alarming. ${ }^{19}$ Long-term use of this drug as a first-line empirical treatment for uncomplicated UTIs in different countries is probably among the main reasons for the emerging resistance. ${ }^{19}$ This indicates the urgent need for an up-to-date evaluation of its effectiveness (resistance profile) against circulating strains of uropathogens, which could restrict its utility, but should allow better use of more suitable candidates as empirical therapy.

The number of resistant strains of $E$. coli to gentamicin and piperacillin increased in Ha'il in the 5-year period of the study (2015-2019), and this rise was reported previously in other regions of Saudi Arabia. ${ }^{19}$ Emergence of resistant strains of $E$. coli to gentamicin might be linked to the unguided usage of empirical therapy for patients with high risk of infection and its complications, such as pregnant patients and children. ${ }^{38}$ This suggestion may explain the high resistance pattern among $E$. coli isolates to gentamycin in this study, as the majority of the patient cohort were pregnant women (and children). The tested Gramnegative isolates recovered from UTI patients in Ha'il showed great sensitivity to amikacin, and this 
outcome is matched with data of a similar investigation in Al-Jouf, Saudi Arabia. ${ }^{39}$ Accordingly, amikacin is one of the promising candidates to be considered for use as a first-line drug for treating patients with UTIs in Ha'il and Al-Jouf regions of Saudi as well as other areas with similar trends.

The emergence of ESBL-producing stains is linked to hospital-acquired UTIs, due to possible initial inappropriate antibiotic therapy, leading to high mortality rate. ${ }^{40}$ This could explain the relatively low number of observed ESBLproducing bacteria in the current study because the majority of the cohort were outpatients. Unsurprisingly, ESBLproducing E. coli and K. pneumoniae isolates showed higher levels of resistance to most antibiotics (ampicillin, ciprofloxacin, gentamicin, nitrofurantoin, piperacillin, tigecycline and trimethoprim/sulfamethoxazole) compared to non-ESBL producing isolates, in agreement with reported reduction in antibiotic susceptibility. ${ }^{41}$ A previous report demonstrated that ESBL-producing E. coli were resistant to gentamicin, piperacillin and ciprofloxacin in Riyadh. ${ }^{42}$ In contrast, over the period of this investigation, Gram-negative UTI isolates (ESBL and non-ESBL producing) exhibited low levels of resistance to cefoxitin, imipenem and meropenem. In line with studies conducted in Riyadh, ${ }^{19}$ the majority of E. coli strains were sensitive to imipenem and meropenem. These findings suggest that carbapenems remain the drugs of choice for treating UTIs caused by multi-drug-resistant (MDR) bacteria in Ha'il.

Gram-positive cocci, including $S$. agalactiae and E. faecalis, accounted for almost a third of UTI cases in this study. This finding is similar to trends in the southern part of Saudi Arabia (Al-Baha), where strains of these species were identified among common causative microorganisms of UTIs. ${ }^{10}$ In line with previous report, ${ }^{43}$ VRE exhibited high resistance to majority of tested antibiotics including trimethoprim/sulfamethoxazole, similar to non-VRE. The majority of Gram-positive uropathogens presented with decent sensitivity to linezolid, suggesting the real need for considering the potential use of this drug as a first drug of choice treating UTIs caused by Gram- positive in Ha'il.

It is worth noting that all urine samples were requested from symptomatic patients after their visit to the hospital, and the nature of collection could indicate possible indirect exclusion of asymptomatic UTI patients. Consequently, it would be useful to investigate the prevalence and antibiotic sensitivity patterns of bacterial isolates from asymptomatic UTI patients in the same region and link the identified trends with the current findings to enable any possible correlation and interpretation. In addition, as the majority of positive urine samples belong to outpatients, this investigation is more likely to represent communityacquired rather than hospital-acquired infections. Furthermore, the restricted access to the clinical data, including date of admission and discharge and risk factors (eg, the use of catheters), makes drawing correlations between bacterial resistance and length of hospitalisation a challenging task.

\section{Conclusion}

Prescribing antibiotics based on sensitivity testing remains the most accurate method, which contributes to controlling/tackling the spread of AMR. However, empirical usage of antibiotics is necessary in serious or urgent cases, which should be performed rationally based on recent and local surveillance data. The current surveillance study analysed data collected over a 5-year period (2015-2019) in the Ha'il region and revealed that the majority of Gram-negative uropathogens, including ESBL, were resistant to trimethoprim/sulfamethoxazole while amikacin, cefoxitin, imipenem and meropenem were highly effective in vitro. In contrast, Gram-positive, including VRE, were sensitive to linezolid. These findings indicate the need for replacement of current first-line antibiotics with more effective alternatives, such as meropenem, amikacin or linezolid. Observed variations in resistance profiles among strains of a single bacterium over time and in different locations demonstrate the need for conducting frequent surveillance studies nationally and locally for more effective monitoring of AMR.

\section{Abbreviations}

AMR, antimicrobial resistance; UTI, urinary tract infection; ESBL, extended-spectrum $\beta$-lactamase; MRSA, methicillin-resistant Staphylococcus aureus; MSSA, methicillin-sensitive Staphylococcus aureus; VRE, vancomycin-resistant enterococci.

\section{Data Sharing Statement}

All data generated in this study are included in this published article.

\section{Author Contributions}

Conception and design; AB and AS. All authors made substantial contributions to acquisition and analysis of data; took part in writing the article or revising it critically for important intellectual content; agreed on the journal to which the article will be submitted; provided their final 
approval of the version to be published; and agreed to be accountable for all aspects of the work.

\section{Funding}

This study was funded by the Scientific Research Deanship at the University of Ha'il, Saudi Arabia, through project reference number: BA-2021.

\section{Disclosure}

The authors declare that there are no conflicts of interest.

\section{References}

1. Flores-Mireles AL, Walker JN, Caparon M, Hultgren SJ. Urinary tract infections: epidemiology, mechanisms of infection and treatment options. Nat Rev Microbiol. 2015;13(5):269-284. doi:10.1038/ nrmicro3432

2. Sobel JD, Kaye D. Urinary Tract Infections. In: Mandell Gl, Bennett JC, Dolin R., editors. Mandell, Douglas, and Bennett's: Principles and Practice of Infectious Diseases. Elsevier; PA, USA. 2014:957-985.

3. Caterino JM, Ting SA, Sisbarro SG, Espinola JA, Camargo CA Jr. Age, nursing home residence, and presentation of urinary tract infection in U.S. emergency departments, 2001-2008. Acad Emerg Med. 2012;19(10):1173-1180. doi:10.1111/j.1553-2712.2012.01452.x

4. François M, Hanslik T, Dervaux B, et al. The economic burden of urinary tract infections in women visiting general practices in France: a cross-sectional survey. BMC Health Serv Res. 2016;16(1):365. doi:10.1186/s12913-016-1620-2

5. Alanazi MQ, Al-Jeraisy MI, Salam M. Prevalence and predictors of antibiotic prescription errors in an emergency department, Central Saudi Arabia. Drug Healthc Patient Saf. 2015;7:103-111. doi:10.2147/DHPS.S83770

6. Alanazi MQ. An evaluation of community-acquired urinary tract infection and appropriateness of treatment in an emergency department in Saudi Arabia. Ther Clin Risk Manag. 2018;14:2363-2373. doi:10.2147/tcrm.S178855

7. Kang CI, Kim J, Park DW, et al. Clinical practice guidelines for the antibiotic treatment of community-acquired urinary tract infections. Infect Chemother. 2018;50(1):67-100. doi:10.3947/ic.2018.50.1.67

8. Onanuga A, Awhowho GO. Antimicrobial resistance of Staphylococcus aureus strains from patients with urinary tract infections in Yenagoa, Nigeria. J Pharm Bioallied Sci. 2012;4(3):226-230. doi:10.4103/0975-7406.99058

9. Alsohaim SIA, Bawadikji AA, Elkalmi R, Mahmud MIA-DM, Hassali MA. Relationship between antimicrobial prescribing and antimicrobial resistance among UTI patients at Buraidah Central Hospital, Saudi Arabia. J Pharm Bioallied Sci. 2019;11(2):162-169. doi:10.4103/jpbs.JPBS_217_18

10. Alzahrani M, Ali M, Anwar S. Bacteria causing urinary tract infections and its antibiotic susceptibility pattern at tertiary hospital in Al-Baha region, Saudi Arabia: a retrospective study. J Pharm Bioallied Sci. 2020;12(4):449-456. doi:10.4103/jpbs.Jpbs_294_19

11. Abejew AA, Denboba AA, Mekonnen AG. Prevalence and antibiotic resistance pattern of urinary tract bacterial infections in Dessie area, North-East Ethiopia. BMC Res Notes. 2014;7(1):687. doi:10.1186/ 1756-0500-7-687

12. Parsonage B, Hagglund PK, Keogh L, Wheelhouse N, Brown RE, Dancer SJ. Control of Antimicrobial resistance requires an ethical approach. Front Microbiol. 2017;8:2124. doi:10.3389/ fmicb.2017.02124
13. Huang ES, Stafford RS. National patterns in the treatment of urinary tract infections in women by ambulatory care physicians. Arch Intern Med. 2002;162(1):41-47. doi:10.1001/archinte.162.1.41

14. Zhanel GG, Karlowsky JA, Harding GK, et al.; The Canadian Urinary Isolate Study Group. A Canadian national surveillance study of urinary tract isolates from outpatients: comparison of the activities of trimethoprim-sulfamethoxazole, ampicillin, mecillinam, nitrofurantoin, and ciprofloxacin. Antimicrob Agents Chemother. 2000;44(4):1089-1092. doi:10.1128/aac.44.4.1089-1092.2000

15. Gupta K, Scholes D, Stamm WE. Increasing prevalence of antimicrobial resistance among uropathogens causing acute uncomplicated cystitis in women. JAMA. 1999;281(8):736-738. doi:10.1001/jama.281.8.736

16. Zowawi HM. Antimicrobial resistance in Saudi Arabia. An urgent call for an immediate action. Saudi Med J. 2016;37(9):935-940. doi:10.15537/smj.2016.9.16139

17. Faidah HS, Ashshi AM, Abou El-Ella GA, Al-Ghamdi AK, Mohamed AM. Urinary tract infections among pregnant women in Makkah, Saudi Arabia. Biomed Pharmacol J. 2015;6(1):01-07. doi:10.13005/bpj/376

18. Eltahawy AT, Khalaf RMF. Urinary tract infection at a University Hospital in Saudi Arabia: incidence, microbiology, and antimicrobial susceptibility. Ann Saudi Med. 1988;8(4):261-266. doi:10.5144/ 0256-4947.1988.261

19. Alanazi MQ, Alqahtani FY, Aleanizy FS. An evaluation of E. coli in urinary tract infection in emergency department at KAMC in Riyadh, Saudi Arabia: retrospective study. Ann Clin Microbiol Antimicrob. 2018;17(1):3. doi:10.1186/s12941-018-0255-z

20. Al-Tawfiq JA, Rabaan AA, Saunar JV, Bazzi AM. Antimicrobial resistance of gram-negative bacteria: a six-year longitudinal study in a hospital in Saudi Arabia. J Infect Public Health. 2020;13 (5):737-745. doi:10.1016/j.jiph.2020.01.004

21. Funke G, Monnet D, deBernardis C, von Graevenitz A, Freney J. Evaluation of the VITEK 2 system for rapid identification of medically relevant gram-negative rods. J Clin Microbiol. 1998;36 (7):1948-1952. doi:10.1128/jcm.36.7.1948-1952.1998

22. Alqasim A, Abu Jaffal A, Alyousef AA. Prevalence of multidrug resistance and extended-spectrum $\beta$-lactamase carriage of clinical uropathogenic escherichia coli isolates in Riyadh, Saudi Arabia. Int J Microbiol. 2018;2018:3026851. doi:10.1155/2018/3026851

23. Fernandes CJ, Fernandes LA, Collignon P. Cefoxitin resistance as a surrogate marker for the detection of methicillin-resistant Staphylococcus aureus. $J$ Antimicrob Chemother. 2005;55 (4):506-510. doi:10.1093/jac/dki052

24. McEwen SA, Collignon PJ. Antimicrobial Resistance: a One health perspective. Microbiol Spectr. 2018;6(2). doi:10.1128/microbiolspec. ARBA-0009-2017

25. Oladeinde BH, Omoregie R, Olley M, Anunibe JA. Urinary tract infection in a rural community of Nigeria. N Am J Med Sci. 2011;3 (2):75-77. doi:10.4297/najms.2011.375

26. Hummers-Pradier E, Ohse AM, Koch M, Heizmann WR, Kochen MM. Management of urinary tract infections in female general practice patients. Fam Pract. 2005;22(1):71-77. doi:10.1093/ fampra/cmh720

27. De Francesco MA, Ravizzola G, Peroni L, Negrini R, Manca N. Urinary tract infections in Brescia, Italy: etiology of uropathogens and antimicrobial resistance of common uropathogens. Med Sci Monit. 2007;13(6):Br136-44.

28. Bander B, Wael M, Sultan A, Abdulrahman A, Abdullah A, Ali S. Antimicrobial susceptibility of microorganisms causing urinary tract infections in Saudi Arabia. J Infect Dev Ctries. 2018;12(04). doi: $10.3855 /$ jidc. 9517

29. Bitew A, Molalign T, Chanie M. Species distribution and antibiotic susceptibility profile of bacterial uropathogens among patients complaining urinary tract infections. BMC Infect Dis. 2017;17(1):654. doi:10.1186/s12879-017-2743-8 
30. Al Yousef SA, Younis S, Farrag E, Moussa H, Bayoumi FS, Ali AM. Clinical and laboratory profile of urinary tract infections associated with extended spectrum $\beta$-lactamase Producing Escherichia coli and Klebsiella pneumoniae. Ann Clin Lab Sci. 2016;46(4):393-400.

31. Al-Harthi AA, Al-Fifi SH. Antibiotic resistance pattern and empirical therapy for urinary tract infections in children. Saudi Med J. 2008;29 (6):854-858.

32. Glennon J, Ryan PJ, Keane CT, Rees JP. Circumcision and periurethral carriage of Proteus mirabilis in boys. Arch Dis Child. 1988;63 (5):556-557. doi:10.1136/adc.63.5.556

33. Qunibi WY. Urinary Tract Infection. Ann Saudi Med. 1982;2 (1):37-46. doi:10.5144/0256-4947.1982.37

34. Muder RR, Brennen C, Rihs JD, et al. Isolation of Staphylococcus aureus from the urinary tract: association of isolation with symptomatic urinary tract infection and subsequent Staphylococcal Bacteremia. Clin Infect Dis. 2006;42(1):46-50. doi:10.1086/498518

35. Altaie SS, Dryja D. Detection of group B Streptococcus. Comparison of solid and liquid culture media with and without selective antibiotics. Diagn Microbiol Infect Dis. 1994;18(3):141-144. doi:10.1016/0732-8893(94)90082-5

36. Le TP, Miller LG. Empirical therapy for uncomplicated urinary tract infections in an era of increasing antimicrobial resistance: a decision and cost analysis. Clin Infect Dis. 2001;33(5):615-621. doi:10.1086/ 322603

37. Karlowsky JA, Kelly LJ, Thornsberry C, Jones ME, Sahm DF. Trends in antimicrobial resistance among urinary tract infection isolates of Escherichia coli from female outpatients in the United States. Antimicrob Agents Chemother. 2002;46(8):2540-2545. doi:10.1128/ aac.46.8.2540-2545.2002
38. Pépin J, Plamondon M, Lacroix C, Alarie I. Emergence of and risk factors for ciprofloxacin-gentamicin-resistant Escherichia coli urinary tract infections in a region of Quebec. Can J Infect Dis Med Microbiol. 2009;20(4):e163-e168. doi:10.1155/2009/971624

39. Taher I, Almaeen A, Aljourfi H, et al. Surveillance of antibiotic resistance among uropathogens in Aljouf region northern Saudi Arabia. Iran J Microbiol. 2019;11(6):468-477.

40. Melzer M, Petersen I. Mortality following bacteraemic infection caused by extended spectrum beta-lactamase (ESBL) producing E. coli compared to non-ESBL producing E. coli. $J$ Infect. 2007;55 (3):254-259. doi:10.1016/j.jinf.2007.04.007

41. Tulara NK. Nitrofurantoin and fosfomycin for extended spectrum beta-lactamases producing escherichia coli and Klebsiella pneumoniae. J Glob Infect Dis. 2018;10(1):19-21. doi:10.4103/jgid. jgid_72_17

42. Al-Agamy MH, Shibl AM, Hafez MM, Al-Ahdal MN, Memish ZA, Khubnani H. Molecular characteristics of extended-spectrum $\beta$ lactamase-producing Escherichia coli in Riyadh: emergence of CTX-M-15-producing E. coli ST131. Ann Clin Microbiol Antimicrob. 2014;13(1):4. doi:10.1186/1476-0711-13-4

43. Alotaibi FE, Bukhari EE. Emergence of vancomycin-resistant enterococci at a Teaching Hospital, Saudi Arabia. Chin Med J (Engl). 2017;130(3):340-346. doi:10.4103/0366-6999.198923
Infection and Drug Resistance

\section{Publish your work in this journal}

Infection and Drug Resistance is an international, peer-reviewed openaccess journal that focuses on the optimal treatment of infection (bacterial, fungal and viral) and the development and institution of preventive strategies to minimize the development and spread of resistance. The journal is specifically concerned with the epidemiology of

\section{Dovepress}

antibiotic resistance and the mechanisms of resistance development and diffusion in both hospitals and the community. The manuscript management system is completely online and includes a very quick and fair peerreview system, which is all easy to use. Visit http://www.dovepress.com/ testimonials.php to read real quotes from published authors. 